\title{
A pessoa inteligente no mundo social
}

\author{
Eliane Gerk Carneiro ${ }^{1}$ \\ Cilio Rosa Ziviani ${ }^{2}$ \\ Universidade Gama Filho
}

\begin{abstract}
Resumo
O conceito de "inteligência" é revisado e cotejado com a noção de "inteligência social". Os contornos teóricos da pessoa inteligente são demarcados com o objetivo de se estabelecer fronteiras definidas pela relação interpessoal mediada por critérios intersubjetivamente válidos. A pessoa inteligente bem sucedida é discutida, especialmente em seus aspectos sociais. Pesquisas no âmbito da inteligência social com origem em modelos conceituais da Psicologia Social são apresentadas, especialmente os estudos centralizados na competência social e os que procuram centralizar a investigação na forma e na precisão do julgamento do outro. Três estudos empíricos de natureza psicométrica são relatados, o primeiro sobre a definição de inteligência social, o segundo constituindo-se na adaptação de um teste de insight social à realidade brasileira e o terceiro sobre a avaliação da inteligência interpessoal em crianças. Finalmente, discute-se os textos mais recentes da obra de Robert J. Stemberg, especificamente os aspectos da aplicabilidade de sua teoria da inteligência bem sucedida às práticas visando a promoção cognitiva.
\end{abstract}

Palavras-Chave: Inteligência social, Inteligência bem sucedida, Pessoa inteligente.

\section{The Intelligent Person in a Social World}

\section{Summary}

The concept of "intelligence" is reviewed and compared to the notion of "social intelligence". The theoretical contour of the intelligent person are delimited with the aim of establishing boundaries defined by the interpersonal relationship mediated by intersubjectively valid criteria. The successful intelligent person is discussed, particularly in its social aspect. Research on social intelligence stemming from Social Psychology conceptual models are presented, particularly those studies focusing on social competence and those studying the way and the precision of social judgement. Three empirical studies of psychometric nature are reported. The first deals with the definition of social intelligence; the second is dedicated to the translation and adaptation of a social insight test to the Brazilian reality; and the third about the evaluation of interpersonal intelligence in children. Finally, the recent work of Robert J. Stemberg is discussed, specifically issues conceming the aplicability of his theory of the successful intelligence to the practical aspects of cognitive promotion.

Key words: Social Intelligence, Successfull intelligence, Intelligent person.

Um dos maiores sucessos da psicologia do século vinte, segundo alguns autores (Herrstein,1973, Gardner, 1998), foi o estudo da inteligência. Trata-se de um tema que fascina leigos e cientistas e que tem levado à elaboração de centenas de livros e milhares de artigos.

Na presente década houve um grande ímpeto nas teorias dedicadas ao conceito de "inteligência". As principais contribuições estão centradas em dois nomes, Robert J. Sternberg (Y ale) e Howard E. Gardner (Harvard). É importante mencionar, ainda, as contribuições de Salovey (Yale) e Mayer (New Hampshire) e a de Daniel Goleman (Harvard) com a introdução da emoção qualificando o conceito de inteligência. Enquanto no início do século o interesse pela inteligência e sua avaliação centralizava-se no contexto escolar, nos dias de hoje, o enfoque se ampliou. Isto se deve ao fato de que há, em nossos dias, uma necessidade de constante aprendizagem devido ao

\footnotetext{
${ }^{1}$ Endereço para correspondência: Rua Canavieiras. 700. ap 903. Grajaú - SP. CEP 20561-000. Telefone: 021576 5711. email: gerk@hexanetcom.br.

2 Endereço para correspondência: Rua Gustavo Sampaio. 426. ap 205. Leme - SP. CEP 22010-010, Telefone: 0215422844.e-mail: cilio@cilio.com.br.
} 
acelerado avanço científico e tecnológico. Novos conceitos estão sendo trabalhados como os de inteligência social (Thorndike, 1920; Wechsler, 1950; Guilford, 1968;), inteligência prática e inteligência bem sucedida (Sternberg, 1985, 1997a), inteligência interpessoal (Gardner,1994); inteligência emocional (Salovey e Mayer, 1990, Goleman,1996), inteligência moral (Boss, 1994). Este artigo parte de uma breve revisão da literatura neste século e oferece essa revisão como pano de fundo para focalização desses principais autores contemporâneos.

A diversidade de utilização do termo "inteligência social" e de enfoques para estudá-lo reflete o que ocorre nos estudos sobre "inteligência". O termo inteligência tem uma utilização surpreendentemente ampla. Na imprensa popular, quando se escreve sobre este tema, não se presume nenhum conhecimento especializado por parte dos leitores. No campo da Psicologia, o termo inteligência pode assumir diversos significados. Stott \& Ball (1965) destacaram três como sendo os principais: (1) potencialidade mental geneticamente determinada; (2) capacidade para adquirir modos de comportamento novos e mais adequados e (3) habilidade(s) para funcionar, fazer, agir em qualquer momento e situação.

A rigor, não há definição absoluta de "inteligência", acerca da qual haja ampla concordância. Há poucos anos, o autor mais produtivo nessa área no momento (Sternberg, 1995), foi particularmente feliz ao declarar que uma das batalhas em torno do conceito de "inteligência", e pode-se argumentar que é a principal batalha, maior mesmo do que a questão hereditariedade-meio, é justamente acerca do que incluir sob a definição de inteligência. Em grande parte, inteligência é algo criado por nós próprios: uma criação para descrever o fato de que em termos de habilidades adaptativas, algumas pessoas têm mais que outras.

As capacidades práticas são adaptativas, pois todos nós precisamos da capacidade de trabalhar com outras pessoas, da capacidade de discernir aspectos do ambiente no qual estamos inseridos. Concluindo o ponto, Sternberg fala de si próprio: "Supostamente agora estou no pedestal da torre de marfim, mas sem usar minha capacidade prática não permanecerei por aqui muito tempo mais" (1995, p. 78) e finaliza dizendo que, para ele, algo se torna parte da inteligência se este algo for necessário à adaptação.

\section{Inteligência social e a pessoa inteligente}

Inteligência social é um conceito antigo, que entra e sai do cenário das pesquisas sobre as capacidades intelectuais há cerca de 70 anos, desde que Thorndike, em 1920, apresentou o termo, distinguindo-o de outras duas inteligências humanas: abstrata e mecânica. A definição original de Thorndike referia-se à capacidade para compreender e lidar com homens e mulheres e agir com sabedoria nas relações humanas. Na década seguinte o termo foi muito usado pelos psicólogos que buscavam desenvolver instrumentos de avaliação (Thorndike \& Stein, 1937).

Concomitantemente, uma área interessada na forma como as pessoas fazem julgamentos a respeito dos outros foi se desenvolvendo (Vernon, 1933). Neste caso, foram utilizados como métodos para coleta de dados avaliações de si próprio e dos outros e a capacidade para assumir o papel de outro ao responder testes e questionários.

Seria lógico supor que as pesquisas sobre inteligência social e sobre julgamento interpessoal se mantivessem próximas ou mesmo intimamente relacionadas, entretanto isto não ocorreu. A princípio, houve um pouco de uso do mesmo vocabulário, um empréstimo de metodologias e instrumentos, e algumas referências cruzadas. Entretanto, até as relações limitadas iniciais foram 
extintas, passando os interessados em inteligência social e os envolvidos com o julgamento de outros a desenvolver áreas de especialização separadas. Subseqüentemente, a pesquisa sobre inteligência social cresceu e esmoreceu, enquanto que a de julgamento interpessoal se desenvolveu consistentemente para o que é chamado hoje de percepção-de-pessoas, processo interpessoal e percepção social.

A divergência entre inteligência social e percepção-de-pessoa poderia ser atribuída a diversas razões. Primeiro, ao limitadíssimo sucesso experimentado pelos que tentaram construir um teste de inteligência social (O'Sullivan, Guilford \& DeMille, 1965; Thorndike \& Stein, 1937), enquanto que os pesquisadores sobre percepção-de-pessoa encontraram sucesso no aprimoramento e aplicação de suas técnicas (Bronfenbrenner, et al., 1970). A falha dos testes de inteligência social contribuíram, sem dúvida, para a perda periódica de interesse no conceito.

A diferença básica, entretanto, decorre do fato de que a área da inteligência social define-se, segundo a famosa exposição de Cronbach (1958), como uma abordagem correlacional, enquanto os pesquisadores da área da percepção de pessoa utilizam o método experimental, ou seja as pesquisas sobre inteligência social seguem uma abordagem psicométrica enquanto as demais estão na área da Psicologia Social. O próprio Thorndike (1920) propôs um método para reaproximar a abordagem psicométrica da inteligência social e da percepção de pessoa quando indicou a dificuldade de se construírem avaliações de inteligência social, afirmando que seria essencial uma situação genuína com pessoas reais.

Seria possível concluir que a reaproximação não seja realmente necessária porque a inteligência social como conceito e área de pesquisa foi superada pela área mais viável de percepção-de-pessoa. Mas este não é o caso. A pesquisa relativa à inteligência social cresceu, perdeu ímpeto e mais uma vez voltou a crescer, com seus defensores enfatizando a importância das diferenças individuais. Além disso, o crescente interesse em conceitos que parecem análogos ao de inteligência social também é evidente na teoria e na abordagem psicogenética do desenvolvimento cognitivo (Piaget, 1950). São trabalhos como os de Piaget (1932) que, ao analisar dados sobre o desenvolvimento moral, observou a insensibilidade da criança pequena diante dos motivos interiores e das intenções dos outros, os de Piaget e Inhelder (1956) sobre a capacidade infantil para representar a experiência perceptiva de outra pessoa, os de Burns e Cavey (1957), que estudaram a "aptidão empática" de crianças entre três e seis anos, os de Flavell (1961), Borkin e Fry (1968), que investigaram a atividade de assunção de papel (roletaking) incluindo. temas como percepção de pessoa, comunicação entre crianças, inferência interpessoal, leitura de pessoas, e os de Wallach (1963) sobre a construção do conhecimento social.

Em contraste com a abordagem cognitiva, Weinstein (1969) dedicou considerável atenção à competência interpessoal em termos de Psicologia Social Sociológica e teoria de aprendizagem social. Sua estrutura conceitual para competência interpessoal enfatizava processos interativos, os atributos dos atores, e os tipos de aprendizagem considerados importantes na realização de tarefas interpessoais.

Outras pesquisas no âmbito da inteligência social tiveram origem em modelos conceituais da Psicologia Social. Um destes modelos é o que cuida do "comportamento socialmente habilidoso" (Caballo, 1987), englobando pesquisas sobre competência social e sobre habilidades sociais. Tais categorias são tomadas por alguns autores como sinônimos. Caballo, por exemplo, procura integrar aspectos de conteúdo e de funcionalidade nos comportamentos socialmente habilidosos. Quanto ao 
conteúdo, este autor se refere a três dimensões gerais para descrever as habilidades sociais. São elas: a) dimensão pessoal, b) dimensão situacional e c) dimensão comportamental, sendo esta considerada em dois níveis de análise, a dos componentes molares e a dos componentes moleculares.

Os estudos centralizados na competência social são de natureza correlacional e assemelhamse bastante aos da abordagem psicométrica, pois buscam identificar o domínio da inteligência social através de análise fatorial aplicada sobre conjuntos de medidas (Keating, 1973; Ford \& Tisak, 1983). Utilizam-se aqui medidas de auto-avaliação, avaliação de professores, entrevistas, medidas de empatia, maturidade social, nível de desenvolvimento moral, assertividade.

Há, ainda, estudos e pesquisas que procuram centralizar a investigação no "como" as pessoas julgam o outro, englobando a "precisão" do julgamento, as características de personalidade consideradas relevantes no julgamento, e a "capacidade" para assumir o papel do outro. Originaramse, como a categoria precedente, em modelos teóricos da Psicologia Social, focalizando aspectos do processamento cognitivo, como a habilidade de leitura do ambiente social.

Do ponto de vista do julgamento interpessoal, pode-se enfatizar a resolução de problemas interpessoais, a atribuição de causalidade aos fatos ou intencional idade às pessoas, a habilidade de colocar-se no lugar do outro. Este tema foi desenvolvido por Heider (1958), quando tratou da reação ao destino do outro e do estabelecimento da objetividade cognitiva do valor de si e do outro.

O interesse renovado pela inteligência social em si e o interesse em conceitos similares por investigadores com orientações teóricas diferentes sugere a necessidade de uma revisão dos trabalhos nesta área, uma vez que os investigadores em uma área freqüentemente parecem não estar familiarizados com trabalhos similares em outras áreas. A dificuldade em se reconhecer que a inteligência social de uma pessoa pode ser a competência interpessoal de outra pessoa aparentemente está baseada não apenas na diversidade de terminologia como também nas origens teóricas diferentes das diversas abordagens.

A origem dos estudos sobre inteligência social encontra-se na definição de Thorndike (1920) que incluía a idéia de capacidade para: (a) entender os outros e (b) agir ou se comportar sabiamente com relação aos outros. Portanto, Thorndike pode ser interpretado como tendo proporcionado: (a) uma dimensão cognitiva de inteligência social, expressa através da apreciação dos outros sem necessidade de ação da parte do que percebe e (b) uma dimensão comportamental, manifesta em ações bem orientadas para o convívio com os outros. Depois desta definição original, a maioria dos investigadores não alterou sua construção, mas simplesmente aceitaram um teste, na maioria deles o George Washington University Social Intelligence Test de Moss e seus colaboradores (Moss, Hunt, Omwake \& Ronning, 1927; Moss, Hunt \& Omwake, 1949; Moss, Hunt, Omwake \& Woodward, 1955), como uma definição operacional de inteligência social (apud Thorndike \& Stein, 1937). É importante observar-se que os próprios Moss et al. (1927) citaram inteligência social como a "habilidade de lidar com os outros..." (p.l 08) e Hunt (1928) indicou que o teste foi projetado para avaliar uma "...habilidade para lidar com pessoas" (p. 317).

Chapin (1939, 1942), que estava a par do trabalho americano sobre inteligência social, lidou diretamente com a George Washington Scale (1939) e mais tarde desenvolveu sua própria escala de "insight social" (1942). Em sua obra mais antiga, Chapin aceitou a escala de George Washington como um critério de avaliação de inteligência social e validou uma escala de participação social. Baseado nela sua conclusão foi que "...a participação social nos grupos e instituições organizados da 
comunidade é, por si própria, uma avaliação robusta de inteligência social..." (p.165). A Escala de Participação Social avalia a dimensão comportamental da inteligência social. Chapin (1942) seguiu desenvolvendo um teste, o Teste de Insight Social, indicador da dimensão cognitiva da inteligência social. Neste último artigo, porém, ele distinguiu explicitamente "insight saciar' de "inteligência saciar' ao indicar que insight social é "...a capacidade de definir... uma dada situação social em termos de comportamento imputado do indivíduo sobre os outros" (p. 215). Esta suposta distinção entre insight social e inteligência social é bem difícil de se compreender e não foi sustentada com dados. Além disto, há que se considerar a dificuldade de se medir "insight", posto que não representa nem uma capacidade nem uma característica pessoal, mas um processo de solução de problemas.

Surgiram outros instrumentos relacionados com a inteligência social incluindo testes ou escalas de empatia e insight. Dymond, Hughes \& Raabe (1952) desenvolveram instrumentos para medir: (a) empatia, que significava "...transposição imaginativa de uma pessoa para o pensamento, sentimento e ação de uma outra" (Dymond, 1950, p. 343) e (b) insight do sujeito, definido por Allport (1937) como sendo "...a relação entre como ele pensa que é com como os outros pensam que ele é" (apud Dymond, 1950, p. 346).

O teste de empatia de Kerr e Speroff $(1947,1951)$ parte de uma definição do termo como a capacidade de alguém se colocar no lugar de uma outra pessoa e antecipar seus comportamentos. Esta abordagem da empatia apresentada por Dymond e por Kerr-Speroff parecem coincidir aceitavelmente com a "compreensão de outros" de Thorndike, mas a versão de insight de DymondAllport, que enfatizava a compreensão de uma pessoa de si própria em relação a como os outros a percebem, cria uma outra perspectiva explícita sobre o conceito de Thorndike.

O interesse em conceitos similares, particularmente na capacidade de assumir papéis aparece exemplificado na teoria e na pesquisa sobre desenvolvimento cognitivo. Apesar de Piaget (1950) ter priorizado os estudos sobre o desenvolvimento de capacidades intelectuais no ambiente não social, tanto Feffer (1959) quanto Flavell e seus colegas (1968) investigaram o curso do desenvolvimento cognitivo em termos de objetos humanos no ambiente interpessoal. Feffer e Suchotliff(1966) propuseram que o conceito de descentralização de Piaget, isto é, a mudança do foco de uma parte do campo perceptual para outra pessoa, pode ser estendido ao conceito interpessoal. Esta capacidade foi avaliada através do teste de assunção de papéis, que requer que $o$ sujeito refocalize as diferentes pessoas apresentadas nas figuras, tornando a narrar a história inicial do ponto de vista de cada um dos personagens. Apesar do teste medir um aspecto da dimensão comportamental de Thorndike, a ênfase de Feffer na atividade cognitiva envolvida num bem sucedido jogo de assumir papéis forneceu uma nova perspectiva para o estudo desta atividade.

Flavell, Botkin e Fry (1968) subdividiram a atividade de assumir papéis em cinco aspectos: (a) o reconhecimento da existência de perspectiva, ou seja, o reconhecimento de que a própria percepção, os próprios pensamentos, ou os próprios sentimentos em uma situação não precisam coincidir com os de outra pessoa; (b) o reconhecimento da necessidade de uma análise da perspectiva do outro e o reconhecimento de que tal análise seria útil para se alcançar uma meta; (c) a capacidade de realmente levar adiante esta análise ou prever com exatidão os atributos das funções relevantes do outro a serem assumidas; (d) a manutenção de cognições produzidas por estas análises em vista de cognições conflitantes representando o seu próprio ponto de vista; e (e) a aplicação destas cognições ao final do processo, como numa comunicação efetiva. 
A consideração destes cinco pontos por Flavell et al inclui a dimensão cognitiva e a comportamental da definição de Thorndike. Pode ser observado mais profundamente que suas investigações da atividade de assumir papéis e de comunicação se baseava em situações interpessoais reais com ênfase em habilidades do sujeito em termos de necessidades

particulares de outros e/ou o que se esperaria que o outro soubesse, ou precisasse saber, se a comunicação fosse efetiva.

Weinstein (1969), ao definir competência interpessoal, observou que ela é "...a capacidade para cumprir tarefas interpessoais", ou seja, "...competência interpessoal diz respeito à capacidade de manipular as respostas dos outros... Competência se refere ao propósito do ator" (p. 755) Apesar desta definição de Weinstein dar grande ênfase ao "lidar com outros" de Thorndike, sua formulação enfatizava a atividade de se assumir papéis de outros e incluía a consideração de modos de interferência sobre o outro. Ele observou que para se tomar o lugar de outro é necessário antecipar seu comportamento, pois somente através da antecipação é possível um controle efetivo. Assim parece que agir sabiamente em termos da definição de Weinstein depende da habilidade da pessoa em antecipar a reação do outro de forma que ele faça o que a pessoa quiser.

Houve, entretanto, usos bem distintos do termo inteligência social que não coincidem com o uso original de Thorndike. Apesar de se referir a Thorndike e usar $o$ termo inteligência social, $o$ Bureau of Public Personnel Administration (1930) descreveu o conceito da seguinte forma:

O essencial é que a pessoa que tem um alto nível de inteligência social seja capaz de conseguir que os outros façam, consistentemente e voluntariamente o que ele quer que elas façam e até gostam de fazê-lo, enquanto que a pessoa sem uma inteligência social considerável não pode obter consistentemente esses resultados ( $p .73$ ).

Esta descrição coincide com o conceito de inteligência maquiavélica utilizado na etologia e na primatologia para caracterizar habilidades de interação social em primatas superiores. O tipo de inteligência denotado por este termo é contrastado com a inteligência técnica ou instrumental (para lidar com objetos). A inteligência maquiavélica, utilizada neste contexto como sinônimo da inteligência social, refere - se à habilidade para fazer e manter alianças com os indivíduos corretos. Cada membro de uma sociedade complexa tem benefícios a ganhar se preservar a estrutura do grupo enquanto simultaneamente manipula os outros. A natureza do sistema criado e mantido pelos primatas superiores exige deles a habilidade de calcular as conseqüências dos seus próprios comportamentos, prever o comportamento dos outros, e equilibrar perdas e ganhos num jogo social semelhante ao xadrez. Este tipo de habilidade também é importante para a sobrevivência do homem no mundo da política e dos negócios (Cronin e Davenport, 1993).

\section{A pessoa inteligente bem sucedida: aspectos sociais}

Particularmente interessante é a proposta de Neisser (1979) sugerindo que a inteligência de uma pessoa é apenas o grau com o qual essa pessoa se parece com o protótipo da "pessoa inteligente". Dado que nenhuma característica única define o protótipo, não poderá haver definição processual adequada de inteligência. Em princípio, o mais adequado é a combinação, em um índice único, de muitas medidas derivadas desse protótipo. A conseqüência é a de que, na prática, muitas das características relevantes ao conceito de "inteligência" simplesmente são impossíveis de medir.

Assim, a preocupação com o que a inteligência é, com a identificação daquilo que a pessoa inteligente tem, enfim, a preocupação de se discutir a real natureza dessa essência mágica ao invés 
de sua distribuição entre as pessoas, tem levado pesquisadores à conclusão de que o conceito de inteligência não pode ser explicitamente definido, não apenas por causa da natureza da inteligência, mas também por causa da natureza dos conceitos (Neisser, 1979). Fundamentando-se no trabalho de Rosch (1978) sobre a estrutura dos conceitos, Neisser acredita que esse tipo de análise aplica-se perfeitamente bem ao conceito de pessoa inteligente, e por meio desse procedimento, por extrapolação, chega-se ao conceito de inteligência propriamente dito.

Mas antes de se estudar as características de uma pessoa inteligente, vale a pena examinar de perto a etimologia do adjetivo "inteligente". A consulta à edição completa do The Random House Dictionary of the English Language (Stein, 1966) mostra que a palavra vem do Latim, intelligent, variante de intellegere, compreender e, literalmente, escolher entre dois (em inglês, choose between). Para tanto, a palavra é composta de intel- (variante de inter) + -lig- (escolher, variante de leg.-, raiz de legere). Da mesma forma, o exame do dicionário etimológico alemão Das Herkunftsworterbuch: Eine Etymologie der deutschen Sprache (Drosdowski et aI., 1963) indica que o adjetivo alemão intelligent vem do latim intelligens, apresentando-se como o particípio presente, acusativo, de intellegere (inter-Iegere), significando perceber algo, tomar algo por verdade, com sentido e compreensão (mil Sinnund Verstand wahrnehmen), ao se "escolher entre dois" (dazwischen wdhlen).

Nesse sentido estrito, a pessoa inteligente é, portanto, aquela capaz de escolher, com sentido e compreensão, entre alternativas. É interessante notar que se aceitamos a tradução da palavra grega nous por "inteligência", "intelecto", tal como os melhores tradutores de Aristóteles costumam fazer, mostra-se como teoricamente produtiva a argumentação do filósofo grego ao discorrer sobre a chance ou fortuna e a espontaneidade ou acaso na parte inicial da Física (Aristotle, $19 S^{\mathrm{b}}$ a $198^{\mathrm{a}}$ ) acerca do tema em pauta.

Aristóteles divide as causas acidentais, ou seja, aquelas que têm um efeito não pretendido, naquelas em que não há deliberação (automaton, espontaneidade, acaso) e naquelas em que há algum grau de escolha racional, inteligente (em cujo caso está a tyche, chance, fortuna). Seres inteligentes são capazes de escolha e essas escolhas sucessivas vão formando sua fortuna, seu destino.

Pode-se portanto chegar a uma conceituação de pessoa inteligente como aquela capaz de escolher com sentido e compreensão entre as oportunidades que a vida lhe oferece e, assim fazendo, ser uma arquiteta parcial na construção de seu próprio destino. Dentro dessa concepção, apresentase como particularmente importante o papel do Outro no processo. Heider (1958), no último capítulo de seu influente texto sobre a psicologia das relações interpessoais, desenvolve a noção da reação que podemos ter ao destino de outra pessoa, tal como por nós percebido. Assim, se consideramos apenas seu valor, isto é, seu caráter positivo ou negativo, toma-se possível distinguir quatro tipos de reação ao destino de outra pessoa.

Embora Heider apenas descreva cada um dos quatro tipos, podemos esquematizá-los da maneira seguinte. No primeiro, o fato do outro passar por uma experiência positiva $(+)$, tal como percebida por nós, mostrar-se como positiva $(+)$ para nós; e no segundo, o fato do outro ter uma experiência negativa (-) ser percebido como igualmente negativo (-) para nós, pressupõe-se, nestes dois primeiros tipos, a concordância, a identificação, o primeiro tipo podendo ser identificado como prazer simpático e o segundo relacionado mesmo à compaixão.

Já os dois últimos tipos, onde se manifestam discordância e antagonismo, são identificados 
como, respectivamente, inveja em um e alegria maliciosa no outro. A inveja refere-se à situação na qual o fato de o outro passar por uma experiência negativa (-) ser positivo (+) para nós, pois dizemos que uma pessoa sente inveja quando menospreza a felicidade de outra; já a alegria maliciosa caracteriza a situação de o outro vivenciar uma situação positiva (+) e essa condição de felicidade percebida no outro ser experimentado por nós como algo negativo (-).

Sem nos alongarmos no detalhamento, pode-se ter aí as condições mínimas básicas para a definição de uma sintaxe da qualidade nas relações interpessoais, dado que as quatro possibilidades simultâneas, (- -), (- +), (+ -) e (++) demarcam a condição sintática para uma lógica das relações intersubjetivas. No capítulo oito, sobre dever e valor, Heider (1958) procura estabelecer teoricamente as bases da objetividade cognitiva do processo de valoração (p. 229) e analisa o conseqüente problema de se atingir um critério intersubjetivamente válido (p. 231). Dimensões, em nosso entender, fundamentais para o norteamento de um programa de estudos sobre a pessoa inteligente no mundo social, especialmente quando este "social" se traduz em competência de se lidar com o outro, ou seja, competência nas relações interpessoais permeadas necessariamente pela condição intersubjetiva.

Assim, entendemos que esse referencial teórico cognitivo servirá de plataforma teórica e metodológica para futuras pesquisas em tomo do conceito de inteligência social, especialmente voltadas para as características idiossincráticas da pessoa inteligente.

Neste ponto cabe mencionarmos um dos trabalhos iniciais de Sternberg, no qual são examinadas as concepções que as pessoas têm de "inteligência" (Stemberg, Conway, Ketron \& Bemstein, 1981) e do qual decorreram conclusões importantes para o norteamento do trabalho futuro. Retomando o modelo da inteligência prototípica de Neisser (1979), Stemberg e seus colaboradores, concluem que as pessoas usam teorias de inteligência implícitas ao avaliarem a própria inteligência e também a inteligência do outro. Essas teorias implícitas são suficientemente similares entre profissionais especialistas de um lado e leigos do outro. Surgiu daí um núcleo comum, encontrado nos sistemas de crença dos indivíduos estudados em todos os grupos. Este núcleo central mostrou-se formado de um fator de solução de problemas, um outro de capacidade verbal, e uma espécie de fator de competência social.

Sternberg \& Smith (1980) localizam suas primeiras contribuições na abordagem socialexperimental baseada na literatura da Psicologia Social sobre comunicação não-verbal. Posteriormente, uma pesquisa foi relatada por Sternberg (1985) na qual algumas técnicas de análise componencial usadas anteriormente nos trabalhos sobre inteligência cognitiva foram aplicadas à análise da inteligência social.

A habilidade de inferir características da pessoa com quem o sujeito está se comunicando tem sido referida como integrante do conceito de "inteligência social" (Ford \& Miura, 1983; Sternberg \& Smith, 1985). Diversas pesquisas trabalharam com o Teste de Sensitividade Não Verbal proposto por Rosenthal e seus colegas (1979). Este teste apresenta fotos de uma única mulher numa variedade de poses. A tarefa do sujeito consiste em decodificar os sinais implícitos que estão sendo emitidos e descobrir qual dentre duas descrições alternativas é mais adequada. Seguindo esta linha, o Teste de Interpretações Sociais (SIT) (Archer e Akert, 1980) apresenta aos sujeitos informações visuais e em alguns casos auditivos, a respeito de uma situação social. Por exemplo, os sujeitos podem ver a foto de uma mulher falando ao telefone e escutar um fragmento da sua conversa. Neste caso, devem inferir se a mulher está falando como um homem ou com 
outra mulher. Em outra situação, os sujeitos devem inferir se um homem e uma mulher apresentados numa foto são estranhos que nunca se falaram antes, conhecidos que já estabeleceram diversas conversações ou amigos há pelo menos seis meses.

Há ainda estudos que se baseiam na existência de inteligências separadas de acordo com localizações cerebrais. Aqui estão incluídos os trabalhos de Howard Gardner sobre as inteligências pessoais e os de Salovey e Mayer (1990) e de Daniel Goleman (1996) sobre a inteligência emocional. São estudos que estão levando a cognição ao campo do sentimento e estão baseados nas novas descobertas sobre a arquitetura emocional do cérebro e a uma teoria diferente e revolucionária sobre a inteligência. Howard Gardner (1995) propôs que não existe um tipo único e monolítico de inteligência mas um amplo espectro de inteligências. Para estudar as inteligências, Gardner baseou-se em dados do desenvolvimento, achados psicométricos, descrições de populações especiais como idiotas sábios e prodígios e especialmente do conhecimento sobre o cérebro obtido a partir de pesquisas recentes nas ciências biológicas.

A teoria das inteligências múltiplas (Gardner, 1995) identifica sete inteligências: lingüística, musical, lógico matemática, espacial, corporal cinestésica, interpessoal e intrapessoal. Num aprimoramento subseqüente deste modelo, a inteligência social é uma categoria superior que cobre quatro categorias discretas: liderança, prudência, mediação de conflitos e análise social (Gardner, 1995).

Já a evolução do corpo teórico de Sternberg destaca o indivíduo, isto é, a pessoa inteligente. Esta evolução poderia ser sintetizada da maneira que se segue, com base nas últimas publicações (Sternberg, 1994; Sternberg, 1995; Sternberg, 1997a; Sternberg, 1997b; Sternberg, 1998 e Sternberg \& Davidson, 1996).

Sternberg acredita que a inteligência bem sucedida é composta das inteligências analítica, criativa e prática. Estas três inteligências se combinam e são aplicadas com o objetivo de se atingir metas importantes. A inteligência bem sucedida é avaliada pela capacidade de se pensar de forma a se desenvolver a excelência pessoal e se obter sucesso no "mundo real". A inteligência bem sucedida, diz Sternberg (1997a), não é apenas uma capacidade cognitiva - é, em grande parte, uma atitude reflexiva sobre a vida, sobre como se está vivendo.

\section{O lado social da inteligência: três estudos}

Com o objetivo principal de estudar o conceito de inteligência em diferentes abordagens teóricas, focalizando especialmente as relações entre aspectos cognitivos, afetivos e sociais na construção do conhecimento, organizamos um seminário de investigações, no Programa de Pós Graduação em Psicologia da Universidade Gama Filho. O plano .geral do trabalho previu três partes: teorias, medidas e aplicações. A fundamentação teórica iniciou-se com a definição de inteligência e de inteligência social, segundo diversas abordagens. A seguir cada uma das abordagens foi aprofundada: os testes psicométricos, com ênfase na teoria fatorial de Guilford, o desenvolvimento cognitivo social através da interação em jogos, a análise componencial da inteligência social na perspectiva de Stemberg, a abordagem dos sistemas simbólicos e as inteligências pessoais, os antecedentes históricos e as críticas ao conceito de inteligência emocional. No que se refere às medidas, foram conduzidos três estudos empíricos, segundo perspectiva psicométrica, que estão relatados a seguir. 


\section{Definição Social de Inteligência Social}

Com o objetivo principal de trabalhar numa primeira abordagem brasileira ao conceito de inteligência social, conduziu-se uma pesquisa exploratória a fim de identificar as teorias implícitas de profissionais cuja atuação se dá na e através da interação social. Stemberg (1985) define teorias implícitas como construções das pessoas que residem em suas mentes. São teorias que precisam ser descobertas e não inventadas, pois elas já existem. A meta neste tipo de pesquisa é descobrir a forma e o conteúdo destas teorias. Exemplo desta abordagem são os estudos de Bruner, Shapiro e Tagiuri (1958) e o de Cantor (1978) a respeito das concepções de inteligência das pessoas comuns. Nestas pesquisas foi solicitado aos sujeitos que 1istassem as características de pessoas inteligentes, daí resultando que tais listas continham características de natureza altamente sociais. Posteriormente, Stemberg e seus colegas (op cit) formalizaram uma investigação do mesmo tipo, na qual solicitaram uma lista de características de pessoas inteligentes academicamente e na vida cotidiana. Aplicando análise fatorial, emergiu um fator que foi denominado "competência social". Os comportamentos que apresentavam altas cargas fatoriais neste fator eram: "aceita os outros como são", "admite erro", "manifesta interesse no mundo como um todo", "está em dia com seus compromissos" e "tem consciência social". Num estudo mais específico sobre "inteligência social", Ford e Miura (apud Stemberg,1985) pediram a estudantes universitários que listassem comportamentos característicos de indivíduos com alta competência social, depois pediram a outros estudantes que classificassem estes comportamentos em grupos. Este estudo revelou quatro dimensões da competência social: a) habilidades pró - sociais, incluindo características como "responder a necessidades dos outros", "é genuinamente interessado nos outros"; b) capacidades de liderança, como "tem habilidade de liderança", "sabe providenciar que as coisas sejam feitas", e "gosta de determinar metas"; c) facilidade social que inclui "está sempre por perto", "é aberto às pessoas", "aprecia atividades sociais"; d) auto eficácia incluindo características como "tem auto conceito positivo", "tem valores e identidade próprios", "possui uma boa visão geral na vida".

No presente estudo, foram questionados 50 profissionais de nível superior que trabalham com pessoas, sendo 13 médicos, 4 psicólogos, 15 professores, 4 fisioterapeutas, 4 assistentes sociais, 3 enfermeiros, 2 advogados, 2 dentistas, 1 nutricionistas e 2 fonoaudiólogas.

Para a coleta de dados, empregou-se a técnica do incidente crítico, proposta por Flannagan (1941). Num questionário composto de 4 itens, perguntou-se aos sujeitos, em primeiro lugar, se eles acreditavam na existência de uma capacidade para lidar com situações sociais; a segunda questão solicitava uma listagem dos atributos característicos de inteligência social, a terceira pedia ao sujeito o relato de um incidente crítico positivo e a última, o relato de um incidente negativo.

Os instrumentos foram respondidos individualmente pedindo-se que registrassem o mais fielmente possível, incidentes positivos e negativos, isto é, acontecimentos particularmente relevantes, procedendo-se a uma análise de conteúdo dos dados coletados em cada questão, constituindo-se assim categorias representativas das dimensões contidas nos relatos.

Para identificar os comportamentos inteligentes, procurou-se, em primeiro lugar, analisar o ambiente social onde eles ocorreram. Os ambientes mais citados para ambos os tipos de incidentes foram (em ordem decrescente de 1Teqüência): 1) ambiente escolar (universidade, escola, faculdade, curso, academia); 2) ambiente hospitalar (CMS, hospital, consultório médico, órgão de inspeção de saúde); 3) ambiente de transporte (ônibus, carro, metrô, táxi); 4) ambiente de rua (rua de lazer, trânsito, praça); 5) ambiente de trabalho (empresa, escritório, sítio); 6) ambiente de serviços 
públicos (banco, repartição pública, justiça); 7) ambiente de lazer (hotel, restaurante, cinema, esporte); 8) ambiente residencial (lar, bairro, condomínio, prédio); 8) ambiente comunitário (favela, creche, comunidade).

A seguir, foram examinadas as descrições tanto do comportamento considerado muito inteligente como do pouco inteligente. Foi elaborado um sistema de categorias para agrupar as respostas. Através de uma análise de conteúdo foram identificadas três dimensões na definição de inteligência social, já referidas por Caballo (1987) e citadas por Del Prette e Del Prette (1996). A primeira dimensão é comportamental e refere-se à classe de comportamentos observáveis, que, no caso dos incidentes positivos foram comportamento pró social, comportamento de liderança e comportamento flexível, e no caso dos incidentes negativos foram: comportamento anti-social, comportamento autoritário, comportamento rígido e comportamento egocêntrico.

A segunda dimensão, denominada pessoal, refere-se às variáveis e processos encobertos do indivíduo relacionados ao comportamento observável como desejos, opiniões, habilidades. Os atributos mais freqüentes nesta categoria foram empatia, assertividade, coerência, fluência verbal, perspicácia, facilidade nas relações interpessoais, acuidade de percepção, capacidade de negociação, boa articulação de idéias, rapidez de raciocínio. Para os incidentes negativos, os atributos foram: mal humor, falta de diálogo, falta de criatividade, insatisfação, incompreensão, pouca habilidade no trato social, intransigência, desinteresse.

A dimensão situacional refere-se a variáveis do contexto em que o comportamento ocorre aludidas genericamente como adequado à situação, são as conseqüências do comportamento inteligente. No caso dos incidentes positivos, estas conseqüências foram: consecução dos objetivos imediatos, manutenção ou melhoria da relação com o outro, manutenção ou melhoria da auto estima, evitação de desconforto físico (quando as interações se dão entre grupos dominados e dominadores). No caso dos incidentes negativos, as conseqüências citadas foram: não atingimento dos objetivos, deterioramento da relação com o outro, sentimento de frustração, egocentrismo e desrespeito ao próximo.

Por último, foram classificados os sentimentos tanto positivos como negativos, provocados no observador pelo autor do comportamento descrito. No caso dos incidentes positivos, os sentimentos provocados foram: admiração, justiça, respeito ao próximo, reflexão, bem estar, e bom humor. No caso dos incidentes negativos, os sentimentos foram: angústia, revolta, incapacidade para ajudar, irritação, decepção.

\section{Uma Medida de Inteligência Social: Estudo da Adaptação do Teste de Insight Social de Chapin à Realidade Brasileira}

Numa primeira aproximação psicométrica ao estudo da inteligência social, realizou-se uma pesquisa com os objetivos de: a) desenvolver estudos com o teste de insight social de F. Stuart Chapin, a fim de verificar sua adequação à realidade brasileira; b) examinar questões de tradução e adaptação de testes para uma cultura diferente; c) fundamentar a construção de um instrumento brasileiro para avaliar um aspecto da inteligência social.

O Teste de Insight Social foi introduzido por Chapin (1942), então responsável pelo Departamento de Sociologia na Universidade de Minnesota. Quarenta e cinco itens preliminares foram reunidos num teste provisório: 4 alternativas de respostas foram preparadas para cada item, 
sendo somente uma delas definida como correta.

Na forma original para pontuar o teste, conta-se o número de respostas corretas tendo peso 3 , e multiplica-se a soma por 3; conta-se o número de respostas corretas com peso 2 e multiplica-se a soma por 2; e conta-se o número de respostas corretas com peso 1 e guarda-se a soma. Finalmente, somam-se os 3 resultados parciais de pontos para se chegar a uma pontuação total. Na tradução preferimos atribuir peso 1 a todos os itens uma vez que não foram estudados os conteúdos das alternativas. Utilizamos a edição de 1993 do Mind Garden, Inc, Pala Alto, California.

Para administração do teste usou-se um livreto de resposta, no qual os 25 itens de situações e as 4 alternativas de resposta para cada situação são listados de forma que o sujeito possa assinalar a resposta apropriada.

A tradução do teste de Insight Social do inglês para o português foi realizada por um tradutor juramentado. A revisão da tradução, por uma professora de inglês, mestranda de psicologia da Universidade Gama Filho, pela coordenadora do projeto e por dois professores do mestrado.

Foi desenvolvida, em primeiro lugar, uma análise semântica a fim de verificar se os itens eram compreensíveis. Foram utilizados como juizes estudantes universitários, em 04 pequenos grupos de 3, numa situação de entrevista em atmosfera de brainstorming. O experimentador apresentou os itens um a um e pediu ao grupo para reproduzi-Ias com suas próprias palavras. Os mesmos estudantes de graduação de Psicologia comunicaram as impressões sobre cada item, a forma como o abordaram e realizaram, os processos utilizados e as facilidades e dificuldades que encontram. Os resultados foram utilizados na reformulação dos itens.

Num segundo momento, procedeu-se a uma análise de conteúdo. Esta análise foi feita por juizes, peritos na área do construto em questão representados por professores de Psicologia que avaliaram se cada item realmente se referia ao fator investigado (Brislin, 1980). A classificação dos itens por conteúdo utilizou três categorias, a saber: julgamento do comportamento do outro (externo), julgamento do próprio comportamento (interno) e opinião própria do respondente.

Aplicou-se, então, o teste em 41 alunos acima de 14 anos de uma escola bilingüe (inglês e português) em suas duas versões: inglês e português. A adequação da tradução foi verificada através do teste de diferença entre proporções para esclarecer a seguinte questão: A proporção de estudantes bilíngües que acertam o item em inglês é igual a proporção dos que acertam em português? $\mathrm{O}$ resultado apresentou estatísticas "Z" altamente significativas, relativas a diferenças entre proporções em tabelas 2 x 2 comparando a dimensão inglês / português com a dimensão acerto/erro. Os resultados variaram de $Z=3,76$ (mínimo) a $Z=9,18$ (máximo) mostrando a baixa equivalência item a item entre as duas versões.

Utilizando-se 101 sujeitos que responderam o teste em português, foi calculado o (de Cronbach, item a item, tendo os coeficientes variado de 0,39 a 0,49, indicando, portanto, baixa fidedignidade.

Para avaliar a validade de constructo foi efetuada análise fatorial, através do método dos componentes principais dos 25 itens tendo-se identificado 10 fatores, não claramente interpretáveis.

Consideramos este teste "intransportável" da cultura para a qual foi construído para outra. Atribuímos esta interpretação ao fato de ter sido feito apenas a tradução lingüística de seus itens que, embora competente do ponto de vista da correspondência textual, deixou muito a desejar quanto ao aspecto semântico necessariamente ligado à cultura onde o teste original foi concebido e conseqüentemente afastado dos aspectos sociais de nossa cultura, especialmente aqueles relativos à 
dimensão das relações interpessoais.

Verificou-se claramente a presença das principais dimensões referidas por Greenfield (1997) em seu artigo You Can 't Take It With You: Why Ability Assessments Don 't Cross Cultures. Com efeito, nem sempre há concordância entre culturas quanto ao valor de determinada resposta; além disso, muito embora a tradução lingüística tenha sido boa, os mesmos itens não significam a mesma coisa, como indicam os resultados psicométricos.

Adicionalmente, considerou-se o constructo insight social importante como indicador de inteligência ao se referir à dimensão interpessoal no contexto teórico da proposta de múltiplas inteligências (Gardner, 1983; Sternberg, 1997) e a impossibilidade do teste ser meramente traduzido e "adaptado" a nossa cultura, exigindo, ao invés disso, a construção de um novo instrumento.

\section{Avaliação da Inteligência Interpessoal em Crianças}

Com o objetivo de avaliar comportamentos indicativos de inteligência interpessoal, foi construída uma escala cujos itens procuram representar comportamentos característicos de crianças que possuem alto índice desta capacidade.

Para a elaboração inicial dos itens, foram realizadas entrevistas com professores de préescola, além de consulta à literatura especializada (Gardner, 1994, 1995, 1996; Walker \& Foley, 1973; Thorndike \& Stein, 1937; Mayer \& Salovey, 1993). Inicialmente foram elaboradas trinta e cinco (35) afirmativas indicadoras dos comportamentos em questão, que em seguida foram submetidas à avaliação de profissionais considerados peritos no assunto.

Os itens foram julgados em relação à objetividade, clareza e pertinência ao conceito de inteligência interpessoal, colocando-os em ordem dos mais representativos para os menos representativos, eliminando aqueles que não eram referentes ao conceito estudado. Após este julgamento restaram vinte e seis (26) consideradas como consistentes.

A escala inicial apresenta trinta e três (33) itens divididos em seis (06) áreas: cooperação (auxílio para um fim comum); criação e manutenção de sinergia (esforço simultâneo para a realização de uma tarefa); percepção de pessoas (perceber os outros e fazer distinções entre eles); empatia (colocar-se no lugar do outro); relacionamento social (interação com outras pessoas) e interesse social (atenção voltada para os outros).

O estudo psicométrico inicial do instrumento resultou numa escala formada por vinte e cinco itens (25), distribuídos em seis dimensões. Das seis dimensões que compunham a escala original, quatro sofreram a eliminação de alguns itens, sem que representasse uma perda significativa. Não houve perda de nenhum item referente a dimensão empatia e a dimensão Interesse pelos outros foi eliminada.

Os resultados encontrados demonstraram que a forma final da escala, resultante da análise fatorial, é consistente e as dimensões suficientes para a avaliação da inteligência interpessoal.

\section{Conclusão}

Talvez o principal avanço nesta década no que diz respeito ao estudo da inteligência seja a crescente atenção que vem merecendo a "pessoa inteligente de bem com a vida" e essa atenção vem se traduzindo por iniciativas que, à primeira vista, poderão parecer com panacéias perigosamente semelhantes aos recursos fáceis da indústria da auto-ajuda. Esta não é a conclusão a que chegamos. 
Com efeito, a pessoa que possui "inteligência bem sucedida" é "esperta" no que diz respeito à realização de seus objetivos. De que maneira? Sabem como tirar o máximo proveito daquilo que fazem bem e sabem como encontrar meios e modos de contornar suas limitações. Motivadas, controladas, perseverantes e independentes, essas são as pessoas que sabem como progredir na vida.

Mas como "sabem" estas duas coisas tão importantes e decisivas? É neste ponto que começa a enorme diferença entre a literatura da auto-ajuda e os textos revisados aqui. As dimensões que compõem essa nova inteligência são mensuráveis e podem ser desenvolvidas. Justamente porque jamais foi descuidado este aspecto fundamental: o da mensuração (como, por exemplo, no significativo trabalho de Almeida e Morais, 1997). Muito embora a queixa principal da área, no momento, seja justamente acerca de deficiências no campo da medida, nem por isso os principais teóricos descuidaram dela.

Neste sentido, o trabalho mais recente de Sternberg (1997a) acerca de como a inteligência prática e a inteligência criativa, além da inteligência analítica, determinam o sucesso na vida, isto é, de como os dois primeiros aspectos da inteligência são os melhores preditores do sucesso no mundo real, mostra-se firmemente ancorado em pesquisa original desenvolvida durante décadas de mensuração sistemática. Em síntese, a inteligência bem sucedida é mensurável e pode ser desenvolvida.

Além disto, há formas específicas de se desenvolver a inteligência prática e a inteligência criativa, formas que qualquer pessoa poderá usar para aumentar as possibilidades de seu próprio sucesso. Ou seja, a inteligência bem sucedida pode ser ativada, e estudos mostram como a pessoa envolvida em realizações significativas faz com que essa ativação da inteligência prática trabalhe em seu próprio beneficio.

Por outro lado, o desempenho criativo requer não apenas a inteligência criativa mas também atributos específicos de personalidade, especialmente a disposição de assumir riscos e a vontade de superar obstáculos. A pessoa criativa tem visão do futuro e a determinação suficiente para muitas vezes caminhar em descompasso com a maioria, como sintetizado na expressão de "comprar na baixa e vender na alta" ("buying /ow and selling high”), freqüentem ente utilizada por Sternberg em seus trabalhos mais recentes (Lubart, 1994; Sternberg, 1997a; Sternberg \& Lubart, 1995; Sternberg \& Lubart, 1996).

Ser inteligente de forma bem sucedida significa pensar bem de três diferentes maneiras: analítica, criativa e praticamente. O pensamento analítico é exigido na solução de problemas e no julgamento da qualidade das idéias. Mas, para início de conversa, a inteligência criativa é exigida pela boa formulação de problemas e idéias, e a inteligência prática é necessária para se usar essas idéias e sua análise de maneira eficaz no dia a dia da pessoa. Assim, muito embora apenas a inteligência analítica seja freqüentemente valorizada nos testes e na sala de aula, esse tipo de inteligência poderá ser menos útil ao estudante em sua futura vida adulta do que a inteligência prática e a inteligência criativa. Em outras palavras, esses três aspectos da inteligência bem sucedida estão relacionados.

Mas relacionados de uma certa maneira, pois a inteligência bem sucedida é mais eficaz quando equilibra todos os seus três aspectos mencionados, o analítico, o criativo e o prático (Sternberg, 1997a). Assim, é mais importante se saber quando e como usar estes aspectos da inteligência bem sucedida do que simplesmente tê-los à disposição e usá-las importunamente. As pessoas inteligentes bem sucedidas não apenas possuem estas capacidades, elas também, e 
principalmente, refletem cuidadosamente acerca de quando usar determinado aspecto e de como usá-lo eficazmente. Enfim, são freqüentem ente capazes de "insight", pois pensam produtivamente.

Para encerrar, fazemos uma última observação. Uma homenagem ao teórico, geralmente não mencionado na literatura que revisamos aqui, mas .que, não obstante, inventou o conceito, produziu a demonstração e cunhou a palavra "insight" (Einsicht) na primeira grande obra de sua vida de cientista, obra essa que, publicada, o conduziu à diretoria do Instituto de Psicologia da Universidade de Berlim em 1921 (Köhler, 1948). Nesse livro, pode-se ler o seguinte: "Todo teste de inteligência é um teste, não apenas da criatura examinada, mas também do próprio experimentador" (p. 265). Observe-se como o mais produtivo autor de obras sobre a inteligência, no momento volta-se para o mesmo conceito (Sternberg \& Davidson, 1996).

Pois bem, em novembro de 1966, Köhler (1969) pronunciou, a convite da Universidade de Princeton, uma série de quatro conferências. Foram os últimos pronunciamentos do último dos grandes representantes da Psicologia da Gestalt, pois faleceu pouco depois, sem ver as conferências impressas. A última dessas conferências intitulou-se "O que é o pensamento?" (What is thinking?). Inicia dizendo que o pensamento (thinking) se torna uma realização, ou seja, se torna produtivo, quando muda nosso meio ambiente mental por meio da solução de problemas (by solvingproblems) que esse meio oferece. Termina discorrendo sobre o processo do "insight" e seu papel na solução de problemas.

Evidentemente, não é por mero acaso que Sternberg vem culminando sua obra em torno dos mesmos conceitos. Então, houve progresso? Claro. Principalmente no campo da prática. Na maneira de se enfocar produtivamente os problemas. Mas, e no campo da teoria? Bem... nem tanto.

\section{Referências}

Almeida, L. S. \& Morais, M. F. (1997) Programa de Promoção Cognitiva. Barcelos, Portugal: Didálvi.

Aristotle. Physics, 4-6, 195b-198a. Em Barnes, 1. (ed.), The Complete Works of Aristotle: The Revised Oxford Translation. VoI. 1. Princeton, NJ: Princeton University Press, 1991.

Archer, D. \& Akert, R. M. (1980) The encoding of meaning: A test of three theories of social interaction, Sociologicallnquiry, 50, 393-419.

Boss,1. (1994) The autonomy ofmoral inteIligence. Educational Theory, 44,399-416.

Bowers, P. \& London, P. (1965) Developmental corre lates of role-playing ability. Child Development, 36, 499-508.

Bruner, I., Shapiro, D. \& Tagiuri, R. (1958) The meaning ofthe traits in isolation and in combination. In R. Tagiuri \& L. Petrollo ( Eds.) Person Perspection and Interpersonal Behavior (pp.277288).Stanford, C.A.: Stanford University Press.

Bureau of Public Personnel Administration (1930) Partially standardized tests of social intelligence. Public Personnel Studies, 8, 73-79.

Burns, N. \& Cavey, L. (1957) Age differences in empathic ability among children. Canadian Journal of Psychology, 11,227-230.

Caballo, V. E. (1987) Teoria, evaluacionyentrenamiento de Ias habilidades sociales. Valencia: Promolibro.

Cantor, N. (1978) Prototypocality and Personality Judgements. Tese de Doutorado, Stanford 
University.

Chapin, F. S. (1939). Social participation and social intelligence. American Sociological Review, 4, 157-166.

Chapin, F. S. (1942) Preliminary standardization of a social insight scale. American Sociological Review, 7,214-225.

Cronbach, L. 1. (1958) The two disciplines of scientific psychology. American Psychologist, 22-42.

Cronin, B. \& davenport, E. (1993) Social inteIligence. Annual Review of Information Science and Technology (ARIST), 28, 3-44.

Del Prette, A . \& Del Prette, Z. A. P. (1998) Efeitos de uma intervenção sobre a topografia das habilidades social dos professores. Psicologia Escolar e Educacional (no prelo).

Drosdowski, G., Müller, W. \& Scholze-Stubenrecht, W. (1963) (eds.) Das H erkunfisworterbuch. Eine Etymologie der deutschen Sprache. Mannheim: Dudenverlag.

Dymond, R. F., Hughes, A. S. \& Raabe, V. L. (1952) Measurable changes in empathy with age. Journal of Consulting Psychology. 16,202-206.

Feffer, M. H. (1959) The cognitive implications of role taking behavior. Journal of Personality, 27, 152-168.

Feffer, M. H. \& Suchotliff, L. (1966) Decentering implications of social interactions. Journal of Personality and Social Psychology. 1966, 4, 415-422.

Flapan, D. (1968) Children 's Understanding ofSocial Interaction. New York: Columbia University Press.

FlaveIl, 1. H. (1966) Role-taking and communication skilIs in children. Y oung Children, 21, 164177.

FlaveIl,1. H., Botkin, P. J. \& Fry, C. L. (1968) The development of role-taking and communication skills in children. New York: Wiley.

Ford, M. E. \& Tisak, M. S. (1983) A further search for social inteIligence. Journal of Educational Psychology, 75, 196-206.

Gardner, H. (1994) A Criança Pré-Escolar: Como Pensa e Como a Escola Pode Ensiná-la. Porto Alegre: Editora Artes Médicas.

Gardner, H. (1994) Estruturas da Mente: A Teoria das Inteligências Múltiplas. Porto Alegre: Artes Médicas.

Gardner, H. (1995) Inteligências Múltiplas: A Teoria na Prática. Porto Alegre: Editora Artes Médicas.

Gilbert, D. (1969) The young child's awareness of affect. Child Development. 40, 629-639.

Greenfield, P. M. (1997) You can't take it with you: Why ability assessments don't cross cultures. American Psychologist, 52, 10, p. 1115-1124.

Goleman, D. (1996) Inteligência Emocional. Rio de Janeiro: Editora Objetiva.

Guilford,1. D. (1968) Intelligence, Creativity, and their Educational Implications. San Diego: Robert Knapp. 
Heider, F. (1958) The Psychology of Interpersonal Relations. New York: Wiley.

Hunt, T. (1928). The measureament of social ～intelligence. Journal of Applied Psychology, 12, 317-334.

Keating, D. (1978) A search for social intelligence. Journal of Educational P ychology, 70, 218223.

Kerr, W. A. \& Speroff, B. J. (1947) Theempathytest. Chicago: Psychometric Affiliates.

Kerr, W. A . \& Speroff, B. 1. (1951) Manual for empathy testo Chicago: Psychometric Affiliates.

Köhler, W. (1948) The Mentality of Apes. 2nd ed. London: Routledge \& Kegan Paul.

Köhler, W. (1969) The Task of Gestalt Psychology. Princeton, NJ: Princeton University Press.

Lubart, T. I. (1994) Creativity. Em Stemberg, R. 1. (1994) Thinking and Problem Solving. New Y ork: Academic Press, 290-332.

Looft, W. R. (1962) Egocentrism and social interaction over the life span. Psychological Bulletin, 78, 73-92.

McClatchy, V. R. (1929) A theoretical and statistical critique of the concept of social intelligence and

of attempts to measure such a processo Journal of Abnormal and Social Psychology, 24, 217220.

Moos, F. A., Hunt, K. T., Omwake, K. T. \& Ronning, M. M. (1927) Social Intelligence Test. Washington, D. C.: The Center for Psychological Service.

Moos, F. A., Hunt, K.T.\& Omwake, K.T (1949) Manual for the Social Intelligence Test. (Rev. Form) Washington, D. C.: The Center for Psychological Service.

Moos, F. A., Hunt, K. T., Omwake, K. T. \& Woodward, L. G. (1955) Manualfor the George Washington UniversitySeries: Sociallntelligence Test. Washington, D. c.: The Center for Psychological Service.

Neisser, U. (1979) The concept of intelligence. Intelligence, 3, 217-227.

O'Sullivan, M, Guilford, J. P. \& Demille, R. (1965) The measurement of social intelligence. Psychological Laboratory Report. Los Angeles: University of Southem Califomia, 34.

Piaget, 1. (1950) The Psychology of Intelligence. London: Routledge \& Kegan Paul.

Pinter, R. \& Upshall, C. C. (1928) Some results of social intelligence tests. School and Society. 27, 369-370.

Rosch, E. R. (1978) Human categorization. Em N. Warren (ed.), Studies in Cross-Cultural Psychology. London: Academic Press.

Rothenberg, B. B. (1970) Children's social sensitivity and the relashionship to interpersonal competence, intrapersonal comfort, and intellectuallevel. Developmental Psychology, 2, 335350.

Salovey. P. \& Mayer, 1.D. (1990) Emotional intelligence. Imagination, Cognition and Personality, 9, 185-211.

Selman, R. (1971) Conceptual role-taking development in early childhood. Proceedings of the 79th Annual Convention of the American Psychological Association. 
Stein, J. (1966) (ed.) The Random House Dictionary of the English Language. The Unabridged Edition. New Y ork: Random House.

Stemberg, R. (1985) Implicit Theories oflntelligence. Journal of Personality and Social Psychology, 49, 3, 607-627.

Sternberg, R. J. (1992) (ed.) As Capacidades Intelectuais Humanas: Uma Abordagem em Processamento de Iriformações. Porto Alegre: Artes Médicas.

Stemberg, R. 1. (1994) (ed.) Encyclopedia of Human Intelligence. New York: MacMillan.

Sternberg, R. J. (1995) "Skeptic" Magazine interview with Robert Stemberg on "The Bel! Curve". Interview by Frank Miele. Skeptic, 3(3), p. 72-80.

Sternberg, R. 1. (1997a) Successful Intelligence: How Practical and Creative Intelligence Determine Success in Life. New Y ork: Plume Books.

Stemberg, R. J. (1997b) The concept of intelligence and its role in lifelong learning and success. American Psychologist, 52.

Stemberg, R. 1., Conway, B. E., Ketron, J. L. \& Bernstein, M. (1981) People's conceptions of intelligence. Journal of Personality and Social Psychology, 41, 3755.

Stemberg, R.1. \& SMith, C. (1985) Social Intelligence and decoding skills in non verbal communication. Social Cognition, 3, 185-192.

Stemberg, R. J. \& Davidson, J. E. (1996) (eds.) The Nature ofInsight. Cambridge: MIT Press.

Stemberg, R. J. \& Lubart, T. I. (1995) Defying the Crowd: Cultivating Creativity in a World of Conformity. New York: The Free Press.

Stemberg, R. 1. \& Lubart, T. I. (1996) Na investment perspective on creative insight. Em Stemberg, R. 1. \& Davidson, 1. E. (eds.) The Nature of Insight. Cambridge: MIT Press, 535-558.

Stott, L. H. \& Ball, R. S. (1965). Intelligence: A changing concept. Monographs ofthe Society for Research on Child Development,30, 4-45.

Strang, R. (1930) Measures of social intelligence. American Journal ofSociology, 36, 263-269.

Thorndike, R. (1920) Intelligence and its uses. Harper 's Magazine, 140, 227-235.

Vemon, P. E. (1933) Some characteristics of the good judge of personality. Journal of Social Psychology, 4, 42-57.

Wechsler, D. (1950) The measurement and appraisal of adult intelligence (4th ed.). Baltimore: Willians \& Wilkins.

Wedeck, J. (1947) The relationship between personality and "psychological ability". British JournalofPsychology, 37,133-151.

Weinstein, E. A. (1969) The development of interpersonal competence.ln: D. A. Goslin (Ed.), Handbook of Socialization Theory and Research. Chicago: Rand McNally, 753-775. 